\title{
Functional/Semantic Gesture Design Factor Studies on Social Robot for User Experience Design
}

\author{
Seung Eun Chung ${ }^{1}$ and Han Young Ryoo ${ }^{2 *}$ \\ Dept. of Content Convergence, Ewha Womans University, 52, Ewhayeodae-gil, \\ Seodaemun-gu, Seoul 03760, Korea \\ 1ilovechse@ewha.ac.kr, ${ }^{2}$ hyryoo@ewha.ac.kr
}

\begin{abstract}
It is necessary to classify the design factors for the user experience design at a level that causes a difference in the aspect of the user experience. So in this research, the functional/semantic gesture design factors have been derived through case studies and a survey where they were amended to have the level values with which a significant difference was verified in the user experience. The organized functional/semantic gesture design factors from the research are the gestures for concept instruction, behavior description, behavior mimic, and functional performance. The concept instruction attribute has been suggested as 'item's sequential/quantity instructions', and 'directional instructions'. The behavior description attribute has been reorganized to 'usage/situational behavior description', and 'symbolic behavior description'. The behavior mimic attribute has been proposed and remained as 'prototype behavior mimic', 'biological behavior mimic', 'personality behavior mimic'. Lastly, the functional performance attribute has been presented as 'direct behavior performance' and 'proxy behavior performance.
\end{abstract}

Keywords: Social service robot, Gesture design factors, Functional/Semantic gesture

\section{Introduction}

As the robot's gestures work as a means of nonverbal communication within the robot-human interactions, the service robot's gestures are considered as important design factors that represent functions and meanings by amplifying, supplementing and emphasizing the linguistic expressions. In the aspect of the fact that the robots copy human behaviors, studies on the human gestures are being proposed in the NUI field, and the gestures intentionally made to convey a meaning can be divided into the manipulative and the semantic expression gestures. The manipulative gestures are the actual motions that move or rotate objects in the environment while the communicative gestures are intended to deliver a unique meaning and are usually accompanied by verbal communication. The semantic gestures are divided into deictic actions, mimetic actions, modalizing symbols, referential symbols [1]. Similar to this, Karam \& Schraefel have classified the gestures according to the gesture styles that deliver a meaning, into the deictic, manipulative, semantic, and descriptive gestures. The deictic gestures refer to the motions that indicate a specific part or direction and the manipulative gestures refer to the imitative behaviors depending on the present operation method. The communicative gestures are the formal symbolic motions taken to grant arbitrary meanings

Article history:

Received (November 28, 2019), Review Result (January 16, 2020), Accepted (February 25. 2020) 
according to the unit operation functions. The descriptive gestures are the actions that show a text or an object of information by drawing it out and these gestures are connected to the referential symbols [2]. To look further into the sub-contents of these gestures at a more specific level, Choi. et al. have suggested in a study that the conceptual meaning description of a function can be divided into the general concept, directional, quantity variation, my status and symbolic descriptions and the image description regarding a function can be divided into usage behaviors, shape of a moving device and icon descriptions [3][4]. Although various studies suggest essential factors to conceptually categorize the gestures, it is necessary to verify that these concepts are suitable to be used as the classification factors for user experience design.

As the user experience obtained through the robot interface is becoming more important these days, the user experience design researches especially in the social robot design field are being actively published [5]. When the designer selects the design factors in the user experience design, it all has to be considered in the user's perspective; therefore, the design factors should be categorized so that there is a user experience difference [6]. Therefore, the design factors for the gesture design should be categorized at a level that generates a difference in the user experience. Besides, these factors should be established at appropriate attributes and level values for the social robots by categorizing them through the actual cases.

In this study, first, through the case studies in the social service field, the gesture design attributes and level values needed to be considered in the robot design have been derived. Secondly, by conducting a user experience survey with the derived attributes and level values, whether there was a significant difference in the aspect of the user experience was verified.

\section{Case studies to derive functional and semantic gesture design factors}

The classification of the functional and semantic gesture design factors found in the literature are manipulative, deictic, mimetic, modal, referential, semantic, and descriptive attributes [1][2][3][4]. In order to establish the various attributes, proposed in the existing researches for the conceptual classification of the functional and semantic gestures, at a suitable level value for social robots, the actual case studies have been collected and organized. To begin with, the functional and semantic gestures were thoroughly examined in the 63 robot design cases from the social service robots that afford gestures; and a total of 11 robots that operate relatively more various gestures have been selected to be organized in detail.

Then, these robot design attributes were sorted according to the derived attributes keywords in the existing literature. If the concepts were duplicated, the attributes were either integrated or eliminated if there were only a few or no case studies that match certain attributes. Through repeated reviews of the bottom-up and top-down approaches, the functional and semantic gesture design attributes have been summarized as the concept instruction, behavior description, behavior mimicry, functional performance gestures and the detailed subdivision concepts and cases are as follows.

\subsection{Concept instruction}

In the concept instruction gestures, there are item or sequential instructions, location instructions, and scale instructions. First of all, (1) item or sequential instructions are the instructions on the subject item itself and on the sequence of the item. For example, a humantype robot "Pepper" shows a number of items when recommending a dish and puts up an arm according to the quantity (i.e. 1-1 in [Figure 1]). Also, it holds out an arm to point at the 
object while explaining about it (i.e. 1-2 in [Figure 1]). Next, (2) the location instructions are the instructions on the concept related to the spatial directions. Another human-type robot "EMIEW3" illustrates the location of the information center and turns its head with an arm holding out to point at the corresponding direction (i.e. 2-1 in [Figure 1]). Furthermore, it guides people to the stores and turns its body to a certain direction (i.e. 2-2 in [Figure 1]). As "Pepper" has a screen at its torso to show service contents, it will look down to the screen to lead people to watch it too as it runs (i.e. 2-3 in [Figure 1]). For another concept, (3) the scale instructions are the instructions on the concept related to the size or quantity."Robi", another human-type robot, can indicate the scope of ability as it stretch out both arms to the sides(i.e. 3-1 in [Figure 1])."Pepper" can also show how large the audience is by extending its arms to the left and to the right(i.e. 3-2 in [Figure 1]).

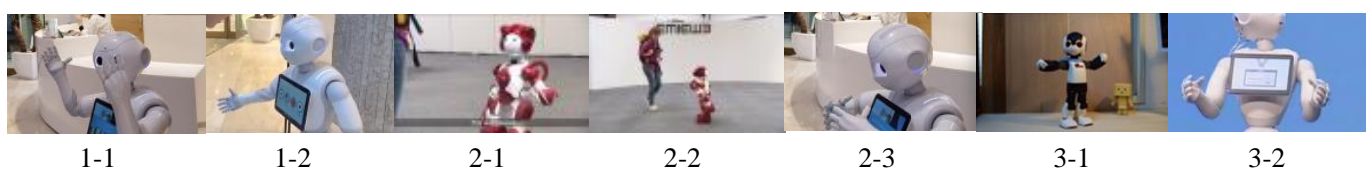

Figure 1. Case image for concept instructional gestures

\subsection{Behavior description}

The usage behavior descriptions, situational behavior descriptions, and symbolic behavior descriptions are the key gestures in the behavior description. To start with, (1) the usage behavior descriptions are the behavior descriptions that use a tool related to a function; such behaviors do not complete the function but it emphasizes the meaning of the function itself. "Pepper" can imitate the action of using a whistle by putting its hand to the mouth and the whistling sound will be made at the same time) (i.e. 1-1 in [Figure 2]). An abstractive robot "JIBO" will shake its tail just like a puppy will do so when he is excited (i.e. 1-2 in [Figure 2]). Second, (2) the situational behavior descriptions are the behaviors that occur in a particular situation and this is to stress the meaning of the situation or to help understand. A human-type robot "Robi" stretches out its torso and shrinks back to describe the cold weather (i.e. 2-1 in [Figure 2]). It also bends its arms and swings them back and forth alternatively to describe the action of taking a walk (i.e. 2-2 in Figure 2). The human-type robot "Pepper" can demonstrate the motion of cutting a steak using a fork and a knife when introducing a steakhouse (i.e. 2-3 in [Figure 2]). Lastly, (3) the symbolic behavior descriptions are the movements that are idiomatically understandable expressions. For example, "Zenbo", an abstractive robot, winks to confirm the order (i.e. 3-1 in [Figure 2]) and "EMIEW3" blows a kiss as it says goodbye to the user (i.e. 3-2 in [Figure 2]).

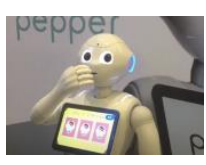

$1-1$

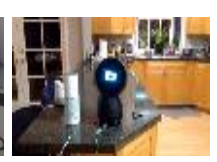

$1-2$

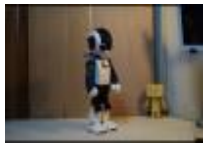

$2-1$

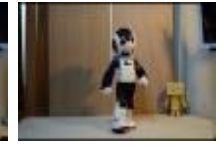

$2-2$

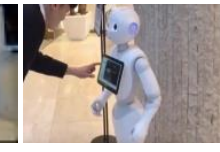

$2-3$

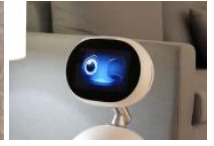

3-1

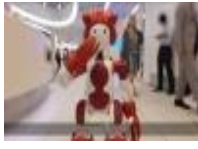

$3-2$

Figure 2. Case Image for behavior depiction gestures

\subsection{Behavior mimicry}


The key behavior mimicry gestures are the prototype behavior mimicry, biological behavior mimicry, and personality behavior mimicry. First, (1) the prototype behavior mimicry is the common sense behavior imitations for a prototype of a person or an animal. For instance, "Nao" which is a human-type robot has the feature of a human and it imitates a person who holds up a V-shape with a hand (i.e. 1-1 in [Figure 3]). An animal type robot "Aibo" has the body shape of a puppy and it shakes its tail to express excitement to mimic a puppy (i.e. 1-2 in [Figure 3]). Besides, (2) the biological behavior mimicry is the common sense behaviors to imitate the characteristics of the robot prototype, such as female, male, a child, an adult, or an elderly. The human-type robot "Robi" can imitate a boy's behavior to reveal a manly image by having the arms bent and placing them on the waist (i.e. $2-1$ in [Figure 3]). Lastly, (3) the personality behavior mimicry is the common sense behaviors that define the robot's unique personality such as extroverted, gentle, or cute. The human-type robot "Robi" is shy when told to be cute and brings its hands to the back and turns its body to the left and to the right (i.e. 3-1 in [Figure 3]). Another human-type robot "EMIEW3" shakes its hand in the air politely to the user who used the information services (i.e. 3-2 in [Figure 3]).

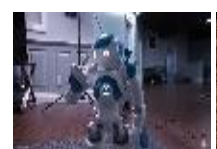

$1-1$

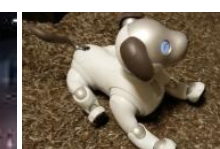

$1-2$

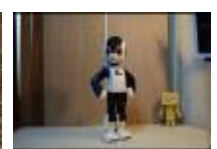

$2-1$

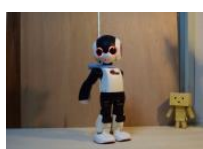

$3-1$

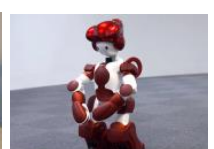

$3-2$

Figure 3. Case image for behavior mimicry gestures

\subsection{Functional performance}

The functional performance gestures can be divided into the direct behavior performance and the proxy behavior performance. First, (1) the direct behavior performance is the direct actions taken to perform a function. For example, a human-type robot "Atom" performs an entertainment function of gymnastics demonstrations by moving its head, legs, arms and/or torso (i.e. 1-1 in [Figure 4]). As for the dancing entertainment functions, an abstractive robot "Cozmo" and the human-type robot "Robi" and "EMIEW3" provide a dance performance by moving and turning its head, arms and torso (i.e. 1-2 in [Figure 4]). The human-type robot "Robi" also offers an entertainment function like a soccer game by participating in the actual soccer game and kicking the ball with its leg (i.e. 1-3 in [Figure 4]). Next, (2) the proxy behavior performance is the actions taken on behalf of the user to perform a function. An animal type robot "Zoomer" can hang a key chain on his tail and deliver (i.e. 2-1 in [Figure 4]). The abstract type robot "Cozmo" can pick up a flat object like a dish with its tongs and carry it (i.e. 2-2 in [Figure 4]).

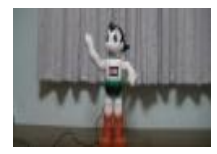

$1-1$

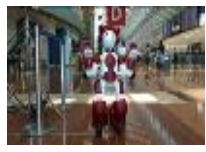

$1-2$

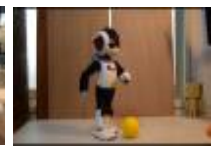

$1-3$

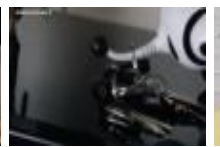

$2-1$

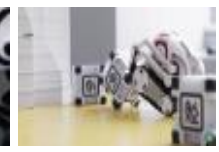

$2-2$

Figure 4. Case image for functional performance gestures

\section{User experience survey and results}

A user experience survey was conducted to analyze the robot gesture design attributes such as concept instructions, behavior descriptions, behavior mimicry, and functional performance. 
The subdivided concepts for one design attributes were considered to be the level values of the design attributes, and these level values were used to check if they show differences towards the user experience; for example, the concept instruction attribute's level values are 'item instructions', 'location instructions' and 'scale instructions'.

For the user experience, the functional and service-wise experience, the interactive experience and the emotional experience [7] were used in the analysis and measured on a 7point Likert scale. The differences in the user experiences were then verified by using the One-Way Analysis of Variance. When there was a difference between the levels, it was verified by the Scheffe test and the findings from this survey are as follows.

To begin with, the average values of the concept instruction gestures, which are divided into 1-1. item/sequential instructions, 1-2. location instructions and 1-3. quantity instructions, have been compared. There was a statistically significant difference in all the instruction gestures towards the functional/service-wise experience, the interactive experience and the emotional experience with the p.value greater than 0.001. According to the Scheffe test, 'item/sequential instructions' and 'quantity instructions' were at the same level of group 'a', while 'location instructions' were at a different level of group ' $b$ '.

Second, the average values of the behavior description gestures, which are divided into 2-1. usage behavior descriptions, 2-2. situational behavior descriptions, and 2-3. symbolic behavior descriptions, were compared. There was no statistically significant difference in the behavior support towards the A) functional /service-wise experience and B) interactive experience. However, a statistically significant difference existed in the difference of the image description towards the C) emotional experience with the p.value greater than 0.01 . The Scheffe test showed that 'usage behavior descriptions' and 'situational behavior descriptions' were at the same level to fall into the group ' $b$ ' while 'symbolic behavior descriptions' were at a different level of group ' $b$ '. Thus, the levels divided into 'usage behavior descriptions', 'situational behavior descriptions' and 'symbolic behavior descriptions' should be altered to 'usage and situational behavior descriptions' and 'symbolic behavior descriptions.

Next, the average values of the behavior mimicry, which are divided into 3-1. prototype behavior mimicry, 3-2. biological behavior mimicry and 3-3. personality behavior mimicry, have been compared. A statistically significant difference in the behavior support in regards to A) functional/service-wise experience and B) interactive experience was found with the p.value greater than 0.001. According to the Scheffe test, 'prototype behavior mimicry' was found in group ' $a$ ', 'biological behavior mimicry' was in group ' $b$ ', and 'personality behavior mimicry' fell into group ' $a b$ '. There was also a statistically significant difference in the behavior support towards C) emotional experience and the p.value was greater than 0.01 . The Scheffe test showed that 'prototype behavior mimicry' and 'personality behavior mimicry' fell into group ' $b$ ' as well as 'biological behavior mimicry'. Since the divided level values all had differences in the functional/service-wise experience, the interactive experience, and the emotional experience, the current level values can remain as is.

Lastly, the average values of the functional performance, which are divided into 4-1. direct action performance and 4-2. proxy action performance, were compared. The difference in the emotional expression towards A) functional/service-wise experience and B) interactive experience showed a statistically significant difference with the p.value greater than 0.01 ; but there was no statistically significant difference in the emotional expression towards $\mathrm{C}$ ) emotional experience. Nonetheless, because a difference between the functional/service-wise 
experience and the interactive experience was verified, there is no need to change the current level values.

Table 1. Evaluation on gesture attributes on user experience

\begin{tabular}{|c|c|c|c|c|c|c|c|c|c|c|c|c|c|c|c|c|c|}
\hline \multicolumn{9}{|c|}{ Concept instruction gesture } & \multicolumn{9}{|c|}{ Behavior mimicry gesture } \\
\hline \multirow{2}{*}{ UX } & \multirow{2}{*}{ No. } & \multirow{2}{*}{ Mean } & \multicolumn{4}{|c|}{ Scheffe } & \multirow{2}{*}{$\mathrm{F}$} & \multirow{2}{*}{$\mathrm{P}$} & \multirow{2}{*}{ UX } & \multirow{2}{*}{ No. } & \multirow{2}{*}{ Mean } & \multicolumn{4}{|c|}{ Scheffe } & \multirow{2}{*}{$\mathrm{F}$} & \multirow{2}{*}{$P$} \\
\hline & & & Group & No. & MD & $\mathrm{P}$ & & & & & & Group & No. & MD & $\mathrm{P}$ & & \\
\hline \multirow{6}{*}{$\mathrm{F}$} & \multirow{2}{*}{$1 \_1$} & \multirow{2}{*}{4.707} & \multirow{2}{*}{ a } & $1 \_2$ & -.407 & .002 & \multirow{6}{*}{15.15} & \multirow{6}{*}{.000} & \multirow{6}{*}{$\mathrm{F}$} & \multirow{2}{*}{ 3_1 } & & & 3_2 & .423 & .008 & & \\
\hline & & & & $1 \_3$ & .207 & .188 & & & & & & a & $3 \_3$ & .264 & .150 & & \\
\hline & & & $\mathrm{h}$ & $1 \_1$ & .407 & .002 & & & & & 4020 & & 3_1 & -.423 & .008 & 500 & \\
\hline & $1 \_2$ & 0.114 & $\mathrm{v}$ & $1 \_3$ & .614 & .000 & & & & $5 z_{2}$ & 4.052 & 0 & 3_3 & -.159 & .500 & 5.00 & .007 \\
\hline & 13 & 4500 & 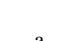 & $1 \_1$ & -.207 & .188 & & & & 3 & 4101 & h & 3_1 & -.264 & .150 & & \\
\hline & $1 \_3$ & 4.500 & $\mathrm{a}$ & 1_2 & -.614 & .000 & & & & 3 & 4.191 & ab & 3_2 & .159 & .500 & & \\
\hline & 1 & 664 & 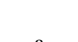 & $1 \_2$ & -.389 & .003 & & & & 3 & 4.450 & 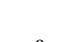 & 3_2 & .461 & .003 & & \\
\hline & 1 & 4.041 & a & $1 \_3$ & .216 & .160 & & & & $3_{-1}$ & 4.459 & a & $3 \_3$ & .280 & .114 & & \\
\hline J & 12 & 5030 & h & 1_1 & .389 & .003 & 1453 & 000 & $J$ & 32 & 3998 & h & 3_1 & -.461 & .003 & 601 & 003 \\
\hline 1 & $1 \_2$ & 5.030 & $\mathrm{D}$ & $1 \_3$ & .605 & .000 & 14.53 & .000 & 1 & $J_{-}^{2}$ & 5.990 & 0 & $3 \_3$ & -.182 & .399 & 0.01 & . \\
\hline & 10 & 1 & & $1 \_1$ & -.216 & .160 & & & & 2 & 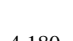 & 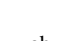 & $3 \_1$ & -.280 & .114 & & \\
\hline & $1 \_3$ & 4.425 & $\mathrm{a}$ & $1 \_2$ & -.605 & .000 & & & & 3 & 4.180 & ab & $3 \_2$ & .182 & .399 & & \\
\hline & & 4306 & & $1 \_2$ & -.402 & .004 & & & & & 561 & $\mathrm{~b}$ & 3_2 & .605 & .000 & & \\
\hline & 1 & 4.396 & $\mathrm{a}$ & $1 \_3$ & .139 & .518 & & & & 3_1 & 4.561 & b & $3 \_3$ & .170 & .477 & & \\
\hline F & 10 & 708 & h & 1_1 & .402 & .004 & 250 & - & $F_{1}$ & 20 & 2057 & 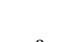 & $3 \_1$ & -.605 & .000 & 007 & ( \\
\hline E & $1 \_2$ & 4.198 & b & $1 \_3$ & .541 & .000 & 25.52 & .000 & E & $3 \_2$ & 3.951 & $\mathrm{a}$ & $3 \_3$ & -.434 & .008 & 9.92 & .000 \\
\hline & 13 & 4257 & 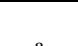 & $1 \_1$ & -.139 & .518 & & & & 33 & 301 & b & $3 \_1$ & -.170 & .477 & & \\
\hline & $1 \_3$ & 4.201 & $\mathrm{a}$ & $1 \_2$ & -.541 & .000 & & & & 3 & 4.391 & D & $3 \_2$ & .434 & .008 & & \\
\hline & & & Behavior & escrip & ion gestu & & & & & & & ctional pe & rforma & ace ges & & & \\
\hline IYY & 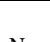 & 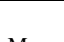 & & & effe & & $\Gamma$ & 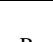 & IYY & N & 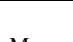 & & Sche & & & $\Gamma$ & 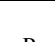 \\
\hline UX & No. & Mean & Group & No. & MD & $\mathrm{P}$ & $\mathrm{F}$ & $P$ & UX & No. & Mean & Group & No. & MD & $\mathrm{P}$ & $\mathrm{F}$ & $\mathrm{P}$ \\
\hline & $2 \_1$ & 4.630 & & & & & & & & 4_1 & 4.802 & & - & & & & \\
\hline F & $2 \_2$ & 4.602 & & & & & .68 & .508 & $\mathrm{~F}$ & 4_2 & 5.146 & & - & & & 7.66 & .006 \\
\hline & $2 \_3$ & 4.500 & & & & & & & 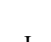 & 4_1 & 4.766 & & - & & & 700 & 005 \\
\hline & $2 \_1$ & 4.621 & & & & & & & 1 & 4_2 & 5.116 & & - & & & 7.90 & .005 \\
\hline I & $2 \_2$ & 4.630 & & & & & 1.95 & .143 & $F_{1}$ & $4 \_1$ & 4.750 & & - & & & 76 & 385 \\
\hline & $2 \_3$ & 4.425 & & & & & & & $\mathrm{E}$ & 4_2 & 4.864 & & - & & & .10 & .385 \\
\hline & 21 & 4577 & h & $2 \_2$ & -.030 & .972 & & & & & & & & & & & \\
\hline & $2-1$ & 4.071 & $\mathrm{v}$ & $2 \_3$ & .320 & .034 & & & & & & & & & & & \\
\hline $\mathrm{F}$ & 22 & 4607 & h & $2 \_1$ & .0230 & .972 & 499 & 007 & & & F: Func & onal / I : & nteract & ion / E & Emotio & & \\
\hline $\mathrm{L}$ & $z_{-}^{2}$ & 4.007 & & $2 \_3$ & .350 & .018 & 4. & (0) & & & & & & & & & \\
\hline & & & & $2 \_1$ & -.320 & .034 & & & & & & & & & & & \\
\hline & $2 \_3$ & 4.257 & $\mathrm{a}$ & $2 \_2$ & -.350 & .018 & & & & & & & & & & & \\
\hline
\end{tabular}

\section{Conclusion}

In this research, the gesture design factors that should be considered in the robot design have been derived first through case studies in the social service field. Then, a user experience survey has been conducted concerning the derived attribute and its level values to verify if there is a significant difference in the aspect of the user experience. The level values that are needed to be adjusted and the ones that can be maintained have been suggested. Although this research was mainly focused on functional and semantic gestures, researches 
on the gestures that express emotions in the aspect of emotional interaction [8] as well as researches on the communicative gestures [9] are significantly arising. In future studies, the base researches on the social robot's design factors are intended to expand through subsequent studies regarding these gestures.

\section{Acknowledgements}

This research was supported by Basic Science Research Program through the National Research Foundation of Korea (NRF) funded by the Ministry of Education (No.2017R1A6A3A11031044).

\section{References}

[1] V. I. Pavlovic, R. Sharma, and T. S. Huang, "Visual interpretation of hand gestures for human-computer interaction: A review,” IEEE Transactions on Pattern Analysis \& Machine Intelligence, vol.19, no.7, pp.677695, (1997) DOI: 0.1109/34.598226

[2] M. Karam and M. C. Schraefel, "A taxonomy of gestures in human computer interactions," Technical Report ECSTR IAM 05-009, Electronics and computer science, university of Southampton, (2005)

[3] J. Choi, T. Kanda, and M. Kim, "Motion design guideline for a humanoid robot," KSDS Conference Proceeding, Korean Society of Design Science, May, (2009)

[4] George Marcus Lloyde and Tomar Geetam Singh, "Comparative Review of Floating-Point Multiplier Systems," International Journal of Hybrid Information Technology, vol.12, no.2, pp.21-48, (2019) DOI:10.21742/IJHIT.2019.12.2.04

[5] S. E. Chung and H. Y. Ryoo, "Social Robot's appearance interface design factor research," International Journal of Artificial Intelligence and Applications for Smart Devices, vol.5, no.2, (2017)

[6] J.J. Garret, “The elements of user experience," New Riders, (2003)

[7] K. Battarbee, "Co-experience: Understanding user experiences in social interaction," Ph.D. dissertation, University of Artand Design Helsinki, (2004)

[8] W. H. Lee, J. W. Park, W. H. Kim, H. S. Lee, and M. J. Chung, "Robot's motivational emotion model with value effectiveness for social human and robot interaction," Journal of Institute of Control, Robotics and Systems, vol.20, no.5, (2014) DOI: 10.5302/J.ICROS.2014.14.9028

[9] J. Jung, T. Kanda, and M. Kim., "Motion design guideline for a humanoid robot," KSDS conference proceeding, Korean Society of Design Science, May, (2009) 
Functional/Semantic Gesture Design Factor Studies on Social Robot for User Experience Design

This page is empty by intention. 